\title{
Back-and-forth systems for fuzzy first-order models
}

\author{
Pilar Dellunde ${ }^{\mathrm{a}, \mathrm{b}, \mathrm{e}}$, Àngel García-Cerdaña ${ }^{\mathrm{a}, \mathrm{c}}$, Carles Noguera ${ }^{\mathrm{d}, *}$ \\ ${ }^{a}$ Artificial Intelligence Research Institute (IIIA-CSIC), Campus de Bellaterra, E-08193 \\ Bellaterra, Catalonia, Spain. \\ ${ }^{b}$ Philosophy Department, Universitat Autònoma de Barcelona, Campus de Bellaterra, \\ E-08193 Bellaterra, Catalonia, Spain. \\ ${ }^{c}$ Information and Communication Technologies Department, University Pompeu Fabra, \\ Tànger, 122-140, E-08018 Barcelona, Catalonia, Spain. \\ ${ }^{d}$ Institute of Information Theory and Automation, Czech Academy of Sciences, Pod \\ vodárenskou věži 4, 18200 Prague, Czech Republic. \\ ${ }^{e}$ Barcelona Graduate School of Mathematics, Campus de Bellaterra, Edifici C, E-08193 \\ Bellaterra, Catalonia, Spain.
}

\begin{abstract}
This paper continues the study of model theory for fuzzy logics by addressing the fundamental issue of classifying models according to their first-order theory. Three different definitions of elementary equivalence for fuzzy first-order models are introduced and separated by suitable counterexamples. We propose several back-and-forth conditions, based both on classical two-sorted structures and on non-classical structures, that are useful to obtain elementary equivalence in particular cases as we illustrate with several examples.
\end{abstract}

Keywords: Mathematical fuzzy logic, first-order fuzzy logics, non-classical logics, elementary equivalence, back-and-forth systems, model theory

\section{Introduction}

Starting from several seminal works (e.g. [33, 31, 24]) and firstly systematized in Petr Hájek's landmark monograph [22, mathematical fuzzy logic (MFL) has been developed as a study of logical systems able to handle graded properties 5 (and related notions of partial truth, vagueness, fuzziness, imprecision, etc.). A

\footnotetext{
* Corresponding author

Email addresses: pilar.dellunde@uab.cat (Pilar Dellunde), angel@iiia.csic.es, angel.garcia@upf.edu (Àngel García-Cerdaña), noguera@utia.cas.cz (Carles Noguera)
} 
considerable amount of papers in the area have resulted in a deep knowledge of, mainly, propositional fuzzy logics as collected in the series of handbooks [8]. However, propositional logics may be seen as insufficient for some purposes because they involve only truth-functional connectives and are not expressive enough to model some important problems in computer science and to provide a satisfactory tool for knowledge representation and analysis of reasoning with graded predicates. There are indeed important areas in fuzzy set theory that deal with first-order notions and thus can be formalized in first-order predicate fuzzy logics such as fuzzy graphs [29], valued preference modelling [19], or fuzzy orders and similarities [2].

Predicate fuzzy logics in the full first-order language with universal and existential quantifiers and an arbitrary stock of functional and relational symbols were already introduced in Hájek's initial works. However, only recently they have become the object of systematic research. The papers [23, 9] give axiomatizations and completeness theorems in a very general framework for graded logics and lay the foundations for an incipient model theory of such logics.

Elementary equivalence is a central notion in classical model theory that allows to classify models by identifying those that validate the same first-order sentences. It was introduced by Tarski 34] and later used by himself and Vaught 25 to study elementary extensions and elementary chains [35]. Inspired by the proof of Cantor's theorem on countable dense linear orderings without endpoints [25, 27, back-and-forth systems of partial isomorphisms were introduced to model theory, and a characterization of elementary equivalence in terms of these systems was introduced in Fraïssé [20, 21]. Independently, the notion was characterized using games in Ehrenfeucht [18. For general surveys on the subject and historical overviews we refer the reader to [1, 5, 17, 26].

In the context of first-order fuzzy logics, the notion of elementary equivalence was defined in [23, Definition 10], where it was used to characterize conservative extensions of theories. A series of papers have followed this definition in various contexts: characterization of strong completeness with respect to models based on a particular class of algebras [7, study of mappings and diagrams [11, ultra- 
product constructions [12, characterization of elementary equivalence in terms of elementarily mappings [14, characterization of elementarily classes as those closed under elementary equivalence and ultraproducts [13], and Löwenheim-

40 Skolem theorems for non-classical logics [15]. An alternative approach considers models of first-order fuzzy logics with evaluated syntax [32, 30]. Finally, an independent, but related, stream of research is that of continuous model theory, in which the underlying logic is essentially Łukasiewicz logic expanded with connectives for each continuous function [6, 4,

The goal of this paper is to contribute to the understanding of elementary equivalence of models of first-order fuzzy logics by focusing on two aspects:

a) considering three different possible generalizations of the classical notion of elementary equivalence to the fuzzy case, that were not distinguished in the previous literature, and

b) providing sufficient back-and-forth conditions to prove elementary equivalence of fuzzy models in particular cases.

The paper is organized as follows: after this introduction, Section 2 presents the necessary preliminaries we need recalling several semantical notions from mathematical fuzzy logic, namely, the algebraic counterpart of extensions of the uninorm logic UL, fuzzy first-order models based on such algebras, and some basic model-theoretic notions. Section 3 defines the notions of elementarily equivalent, filter-strong elementarily equivalent, and strongly elementarily equivalent fuzzy models and separates them with natural counterexamples. Section 4 proposes a classical approach to the problem of finding back-and-forth conditions for elementary equivalence of fuzzy models by treating them as classical two-sorted structures. After this, Section 5 presents a genuinely non-classical approach that, based on a syntactical notion of nested rank, allows to build layered backand-forth systems to prove elementary equivalence of fuzzy models restricted to sentences up to a certain degree of syntactical complexity. Finally, Section 6 65 ends the paper by discussing a straightforward generalization of the results to a much wider framework and some other concluding remarks. 


\section{Preliminaries}

\subsection{Fuzzy first-order models}

Model theory studies mathematical structures using different formal languages. In this section we introduce fuzzy first-order models, the object of our study. On the one hand, they provide the semantics of first-order predicate fuzzy logics. On the other hand, fuzzy first-order models can be seen also as two-sorted classical structures. As a general reference for all the notions of mathematical fuzzy logic that we will use in the paper one can consult the handbook 8 .

As underlying propositional basis for the first-order predicate formalism, we choose a well-established class of propositional fuzzy logics: residuated uninormbased logics (studied in [28]). Such class provides a reasonable framework with several advantages: (1) it contains most of the well-studied particular systems of fuzzy logic that can be found in the literature, (2) it includes weakening-free logics and, hence, it benefits from their modeling power for reasoning with graded predicates as argued in [10], and (3) it retains the properties of associativity and commutativity of the residuated conjunction which simplifies the language and the formulation of many results.

These fuzzy logics can be introduced by means of algebraic semantics based on UL-algebras, that is, algebraic structures in the language $\mathcal{L}=\{\wedge, \vee, \&, \rightarrow$ $, \overline{0}, \overline{1}, \perp, \top\}$ of the form $\boldsymbol{A}=\left\langle A, \wedge^{\boldsymbol{A}}, \vee^{\boldsymbol{A}}, \&^{\boldsymbol{A}}, \rightarrow^{\boldsymbol{A}}, \overline{0}^{\boldsymbol{A}}, \overline{1}^{\boldsymbol{A}}, \perp^{\boldsymbol{A}}, \top^{\boldsymbol{A}}\right\rangle$ such that

- $\left\langle A, \wedge^{\boldsymbol{A}}, \vee^{\boldsymbol{A}}, \perp^{\boldsymbol{A}}, \top^{\boldsymbol{A}}\right\rangle$ is a bounded lattice,

- $\left\langle A, \&^{\boldsymbol{A}}, \overline{1}^{\boldsymbol{A}}\right\rangle$ is a commutative monoid,

- for each $a, b, c \in A$, we have:

$$
\begin{array}{ll}
a \&^{\boldsymbol{A}} b \leq c \text { iff } \quad b \leq a \rightarrow{ }^{\boldsymbol{A}} c, & \text { (residuation) } \\
\left(\left(a \rightarrow^{\boldsymbol{A}} b\right) \wedge \overline{1}^{\boldsymbol{A}}\right) \vee^{\boldsymbol{A}}\left(\left(b \rightarrow^{\boldsymbol{A}} a\right) \wedge^{\boldsymbol{A}} \overline{1}^{\boldsymbol{A}}\right)=\overline{1}^{\boldsymbol{A}} . & \text { (prelinearity) }
\end{array}
$$

90

It is interesting to observe that in such algebras the lattice order can be described in terms of $\rightarrow$ in the following way: $a \leq b$ iff $\left(a \rightarrow \rightarrow^{\boldsymbol{A}} b\right) \wedge^{\boldsymbol{A}} \overline{1}^{\boldsymbol{A}}=\overline{1}^{\boldsymbol{A}}$. 
$\boldsymbol{A}$ is called a UL-chain if its lattice is linearly ordered. If $\boldsymbol{A}$ is defined over the real unit interval $[0,1]$ with its usual order, then it is called a standard ULchain and the operation $\&^{\boldsymbol{A}}$ is a residuated uninorm, that is, a left-continuous binary associative commutative monotonic operation with a neutral element $\overline{1}^{\boldsymbol{A}}$ (which need not coincide with the value 1).

Let $F m_{\mathcal{L}}$ denote the set of propositional formulas written in the language of UL-algebras with a denumerable set of variables and let $\boldsymbol{F} \boldsymbol{m}_{\mathcal{L}}$ be the absolutely free algebra defined on such set. Given a UL-algebra $\boldsymbol{A}$, we say that an $\boldsymbol{A}$ evaluation is a homomorphism from $\boldsymbol{F} \boldsymbol{m}_{\mathcal{L}}$ to $\boldsymbol{A}$. The logic of all UL-algebras is defined by establishing, for each $\Gamma \cup\{\varphi\} \subseteq F m_{\mathcal{L}}, \Gamma \vdash_{\text {UL }} \varphi$ if and only if, for each UL-algebra $\boldsymbol{A}$ and each $\boldsymbol{A}$-evaluation $e$, we have $e(\varphi) \geq \overline{1}^{\boldsymbol{A}}$, whenever $e(\psi) \geq \overline{1}^{\boldsymbol{A}}$ for each $\psi \in \Gamma$. The logic UL is, hence, defined by means the preservation of truth over all UL-algebras, where the notion of truth is taken as belonging to the set of designated elements, or filter, $\mathcal{F}^{\boldsymbol{A}}=\left\{a \in A \mid a \geq \overline{1}^{\boldsymbol{A}}\right\}$. The standard completeness theorem of UL proves that the logic is also complete with respect to its intended semantics: the class of UL-chains defined over $[0,1]$ by residuated uninorms (the standard UL-chains); this justifies the name of UL (uninorm logic).

As we mentioned above, a majority of propositional fuzzy logics can be obtained by extending UL with additional axioms and rules (in a possibly expanded language). A particular kind of expansions (we will refer to them in the paper) is obtained by adding a truth-constant $\bar{r}$ for each value $r$ in a chosen algebra. We will also use some examples of logics satisfying the weakening law, 115 that is, extensions of UL in which, for each algebra $\boldsymbol{A}$ of the logic, $\top^{\boldsymbol{A}}=\overline{1}^{\boldsymbol{A}}$ (hence the filter is the singleton $\mathcal{F}^{\boldsymbol{A}}=\left\{\overline{1}^{\boldsymbol{A}}\right\}$ ) and $\perp^{\boldsymbol{A}}=\overline{0}^{\boldsymbol{A}}$. We will use two well-known examples of such logics with weakening: the Gödel-Dummett logic $\mathrm{G}$ and the Lukasiewicz logic $\mathrm{E}$, respectively defined on the standard UL-chains, $[0,1]_{\mathrm{G}}$ and $[0,1]_{\mathrm{E}}$ given by the following operations: 


$$
\begin{aligned}
a \&^{[0,1]_{\mathrm{G}}} b & =\min \{a, b\}, \\
a \rightarrow^{[0,1]_{\mathrm{G}}} b & = \begin{cases}1, & \text { if } a \leq b, \\
b, & \text { otherwise, }\end{cases} \\
a \&^{[0,1]_{\mathrm{E}}} b & =\max \{0, a+b-1\}, \\
a \rightarrow^{[0,1]_{\mathrm{E}}} b & = \begin{cases}1, & \text { if } a \leq b, \\
1-a+b, & \text { otherwise. }\end{cases}
\end{aligned}
$$

A predicate language $\mathcal{P}$ is a triple $\langle\mathbf{P}, \mathbf{F}$, ar $\rangle$, where $\mathbf{P}$ is a non-empty set of predicate symbols, $\mathbf{F}$ is a set of function symbols, and ar is a function assigning to each symbol a natural number called the arity of the symbol. Let us further fix a denumerable set $V$ whose elements are called object variables. The sets of $\mathcal{P}$-terms, atomic $\mathcal{P}$-formulas, and $\langle\mathcal{L}, \mathcal{P}\rangle$-formulas are defined as in classical logic. A $\mathcal{P}$-structure $\mathbf{M}$ is a pair $\langle\boldsymbol{A}, \mathbf{M}\rangle$ where $\boldsymbol{A}$ is a UL-chain and $\mathbf{M}=$ $\left\langle M,\left\langle P_{\mathbf{M}}\right\rangle_{P \in \mathbf{P}},\left\langle F_{\mathbf{M}}\right\rangle_{F \in \mathbf{F}}\right\rangle$, where $M$ is a non-empty domain; $P_{\mathbf{M}}$ is a function $M^{n} \rightarrow A$, for each $n$-ary predicate symbol $P \in \mathbf{P}$; and $F_{\mathbf{M}}$ is a function $M^{n} \rightarrow$ $M$ for each $n$-ary function symbol $F \in \mathbf{F}$. An $\mathbf{M}$-evaluation of the object variables is a mapping $\mathrm{v}: V \rightarrow M$; by $\mathrm{v}[x \rightarrow a]$ we denote the M-evaluation where $\mathrm{v}[x \rightarrow a](x)=a$ and $\mathrm{v}[x \rightarrow a](y)=\mathrm{v}(y)$ for each object variable $y \neq x$. We define the values of the terms and the truth values of the formulas as:

$$
\begin{array}{rlrl}
\|x\|_{\mathrm{v}}^{\mathbf{M}} & =\mathrm{v}(x), \\
\left\|F\left(t_{1}, \ldots, t_{n}\right)\right\|_{\mathrm{v}}^{\mathbf{M}} & =F_{\mathbf{M}}\left(\left\|t_{1}\right\|_{\mathrm{v}}^{\mathbf{M}}, \ldots,\left\|t_{n}\right\|_{\mathrm{v}}^{\mathbf{M}}\right), & \text { for } F \in \mathbf{F}, \\
\left\|P\left(t_{1}, \ldots, t_{n}\right)\right\|_{\mathrm{v}}^{\mathbf{M}} & =P_{\mathbf{M}}\left(\left\|t_{1}\right\|_{\mathrm{v}}^{\mathbf{M}}, \ldots,\left\|t_{n}\right\|_{\mathrm{v}}^{\mathbf{M}}\right), & \text { for } P \in \mathbf{P}, \\
\left\|\circ\left(\varphi_{1}, \ldots, \varphi_{n}\right)\right\|_{\mathrm{v}}^{\mathbf{M}} & ={ }^{\boldsymbol{A}}\left(\left\|\varphi_{1}\right\|_{\mathrm{v}}^{\mathbf{M}}, \ldots,\left\|\varphi_{n}\right\|_{\mathrm{v}}^{\mathbf{M}}\right), & \text { for } \circ \in \mathcal{L}, \\
\|(\forall x) \varphi\|_{\mathrm{v}}^{\mathbf{M}} & =\inf _{\leq_{A}}\left\{\|\varphi\|_{\mathrm{v}[x \rightarrow a]}^{\mathrm{M}} \mid a \in M\right\}, & \\
\|(\exists x) \varphi\|_{\mathrm{v}}^{\mathbf{M}} & =\sup _{\leq_{\mathbf{A}}\left\{\|\varphi\|_{\mathrm{v}[x \rightarrow a]}^{\mathrm{M}} \mid a \in M\right\} .}
\end{array}
$$

If the infimum or supremum does not exist, the corresponding value is undefined. We say that $\mathbf{M}$ is a model (or $\mathcal{P}$-model if we want to stress the predicate language) if it is safe, that is, if $\|\varphi\|_{\mathrm{v}}^{\mathrm{M}}$ is defined for each $\mathcal{P}$-formula $\varphi$ and each M-evaluation v. Formulas without free variables are called sentences. Observe that if $\varphi$ is a sentence, then its value does not depend on a particular M-evaluation; we denote its value as $\|\varphi\|_{\mathbf{M}}^{A}$. 
The notion of fuzzy first-order model just defined allows to provide a semantics for the first-order counterparts of the propositional fuzzy logics we have mentioned above. One can give axiomatizations and corresponding completeness theorems for such first-order fuzzy logics; however, this is not the topic of the present paper in which we concentrate on the study of the models.

The connection with two-sorted languages and structures is quite straightforward. We have described it in formal details in [15, Section 7]. The idea is that each fuzzy first-order structure $\langle\boldsymbol{A}, \mathbf{M}\rangle$ can be seen as a two-sorted structure: (1) the first sort contains the elements of $A$ and has the algebraic operations as functionals applied to the first sort and taking values in the same sort, (2) the second sort contains the elements of the domain $M$ and has the functional symbols of the predicate language acting as inner functionals of the second sort while the predicate symbols are functionals that apply to elements of the second sort and return values in the first sort. The two-sorted language has an equality symbol for each sort, which makes it expressive enough to describe the fuzzy structure to a big extent. In particular, one can build two-sorted formulas to express truth values of formulas of our predicate languages for fuzzy logics.

Finally, let us recall several notions of mappings and homomorphisms between fuzzy first-order structures that will be used in the paper. Let $\boldsymbol{A}$ and $\boldsymbol{B}$ be UL-chains and let $\langle\boldsymbol{A}, \mathbf{M}\rangle$ and $\langle\boldsymbol{B}, \mathbf{N}\rangle$ be $\mathcal{P}$-structures. Let $f$ be a mapping 160 from $A$ to $B$, and $g$ be a mapping from $M$ to $N$. The pair $\langle f, g\rangle$ is said to be a mapping from $\langle\boldsymbol{A}, \mathbf{M}\rangle$ to $\langle\boldsymbol{B}, \mathbf{N}\rangle$. If $f$ preserves all the existing infima and suprema, then $\langle f, g\rangle$ is called a $\sigma$-mapping. A mapping $\langle f, g\rangle$ is said to be a strong homomorphism if:

1) $f$ is a homomorphism of UL-algebras.

2) $g: M \rightarrow N$ is a homomorphism between the algebraic reducts of the firstorder structures, that is, for every $n$-ary function symbol $F \in \mathcal{P}$ and $d_{1}, \ldots, d_{n} \in M$

$$
g\left(F_{\mathbf{M}}\left(d_{1}, \ldots, d_{n}\right)\right)=F_{\mathbf{N}}\left(g\left(d_{1}\right), \ldots, g\left(d_{n}\right)\right) .
$$


3) For every $n$-ary predicate symbol $P \in \mathcal{P}$ and $d_{1}, \ldots, d_{n} \in M$,

$$
f\left(P_{\mathbf{M}}\left(d_{1}, \ldots, d_{n}\right)\right)=P_{\mathbf{N}}\left(g\left(d_{1}\right), \ldots, g\left(d_{n}\right)\right) .
$$

We say that a strong homomorphism $\langle f, g\rangle$ is an elementary homomorphism if for every formula $\varphi\left(x_{1}, \ldots, x_{n}\right)$, and $d_{1}, \ldots, d_{n} \in M$,

$$
f\left(\left\|\varphi\left(d_{1}, \ldots, d_{n}\right)\right\|_{\mathbf{M}}^{\boldsymbol{A}}\right)=\left\|\varphi\left(g\left(d_{1}\right), \ldots, g\left(d_{n}\right)\right)\right\|_{\mathbf{N}}^{B}
$$

Now we introduce the notions of partial isomorphism and of back-and-forth systems between two fuzzy first-order structures. These notions are not new but a reformulation of the usual definitions for classical two-sorted structures.

Definition 1 (Partial Mapping). Let $\langle\boldsymbol{A}, \mathbf{M}\rangle$ and $\langle\boldsymbol{B}, \mathbf{N}\rangle$ be $\mathcal{P}$-models. Let $p$ be a partial mapping from $A$ to $B$, and $r$ be a partial mapping from $M$ to $N$. The pair $\langle p, r\rangle$ is said to be a partial mapping from $\langle\boldsymbol{A}, \mathbf{M}\rangle$ to $\langle\boldsymbol{B}, \mathbf{N}\rangle$.

Definition 2 (Partial Isomorphism). A partial mapping $\langle p, r\rangle$ is a partial isomorphism from $\langle\boldsymbol{A}, \mathbf{M}\rangle$ to $\langle\boldsymbol{B}, \mathbf{N}\rangle$ if

1. $p$ and $r$ are injective,

2. for every $n$-ary connective $\lambda \in \mathcal{L}$, and every $a_{1}, \ldots, a_{n} \in A$, such that $a_{1}, \ldots, a_{n}, \lambda^{\mathbf{A}}\left(a_{1}, \ldots, a_{n}\right) \in \operatorname{dom}(p)$,

$$
p\left(\lambda^{\boldsymbol{A}}\left(a_{1}, \ldots, a_{n}\right)\right)=\lambda^{\boldsymbol{B}}\left(p\left(a_{1}\right), \ldots, p\left(a_{n}\right)\right),
$$

3. for every $n$-ary functional symbol $F \in \mathcal{P}$ and every $d_{1}, \ldots, d_{n} \in M$ such that $d_{1}, \ldots, d_{n}, F_{\mathbf{M}}\left(d_{1}, \ldots, d_{n}\right) \in \operatorname{dom}(r)$,

$$
r\left(F_{\mathbf{M}}\left(d_{1}, \ldots d_{n}\right)\right)=F_{\mathbf{N}}\left(r\left(d_{1}\right), \ldots, r\left(d_{n}\right)\right)
$$

4. For every $n$-ary predicate symbol $R \in \mathcal{P}$ and $d_{1}, \ldots, d_{n} \in M$ such that $d_{1}, \ldots, d_{n} \in \operatorname{dom}(r)$,

$$
p\left(R_{\mathbf{M}}\left(d_{1}, \ldots, d_{n}\right)\right)=R_{\mathbf{N}}\left(r\left(d_{1}\right), \ldots, r\left(d_{n}\right)\right) .
$$


175 Remark 3. Observe that from the second condition of Definition 2 it follows that $p\left(\overline{0}^{\boldsymbol{A}}\right)=\overline{0}^{\boldsymbol{B}}$ and $p\left(\overline{1}^{\boldsymbol{A}}\right)=\overline{1}^{\boldsymbol{B}}$, whenever such elements are in the domain of p. This, together with the first condition, implies that for every $a \in \operatorname{dom}(p), a \in$ $\mathcal{F}^{\boldsymbol{A}}$ if and only if $p(a) \in \mathcal{F}^{\boldsymbol{B}}$. Observe, finally, that clause 4 states implicitly that for every $n$-ary relational $R$, and every $d_{1}, \ldots, d_{n} \in M$, such that $d_{1}, \ldots, d_{n} \in$ $180 \operatorname{dom}(r)$, it holds that $R_{\mathbf{M}}\left(d_{1}, \ldots, d_{n}\right) \in \operatorname{dom}(p)$.

An example of a partial isomorphism is $\langle\emptyset, \emptyset\rangle$. A less trivial example is described as follows:

Example 4. Let $\mathcal{P}$ be a predicate language with a binary predicate symbol $R$ and a binary functional symbol $F$. Consider the $\mathcal{P}$-models $\langle\boldsymbol{A}, \mathbf{M}\rangle$, and $\langle\boldsymbol{A}, \mathbf{N}\rangle$, where $M=N=\{0,1,2\}$, and $\boldsymbol{A}$ is the UL-chain defined on $\left\{0, \frac{1}{2}, 1\right\}$ by the monoidal operation given by the table:

\begin{tabular}{|c|ccc|}
\hline$*$ & 0 & $\frac{1}{2}$ & 1 \\
\hline 0 & 0 & 0 & 0 \\
$\frac{1}{2}$ & 0 & $\frac{1}{2}$ & 1 \\
1 & 0 & 1 & 1 \\
\hline
\end{tabular}

Observe that $\mathcal{F}^{\mathbf{A}}=\left\{\frac{1}{2}, 1\right\}$ (because the constant $\overline{1}$ is interpreted as the neutral element $\left.\frac{1}{2}\right)$. The interpretation of $F$ in both models is the operation $\oplus$ defined as follows:

\begin{tabular}{|l|lll|}
\hline$\oplus$ & 0 & 1 & 2 \\
\hline 0 & 0 & 1 & 2 \\
1 & 1 & 2 & 0 \\
2 & 2 & 0 & 1 \\
\hline
\end{tabular}

$R_{\mathrm{M}}$ is defined by the matrix:

$$
\left(\begin{array}{ccc}
0 & 1 & 1 \\
\frac{1}{2} & \frac{1}{2} & 1 \\
1 & 1 & \frac{1}{2}
\end{array}\right)
$$


and $R_{\mathbf{N}}$ is defined by the matrix:

$$
\left(\begin{array}{ccc}
0 & 1 & 0 \\
1 & \frac{1}{2} & 1 \\
0 & 1 & \frac{1}{2}
\end{array}\right)
$$

Now consider the partial mapping $\langle p, r\rangle$ from $\langle\boldsymbol{A}, \mathbf{M}\rangle$ to $\langle\boldsymbol{A}, \mathbf{N}\rangle$, where $p=$ $I d_{A}$ and $r=\{\langle 1,2\rangle,\langle 2,1\rangle\}$. It is easy to check that $\langle p, r\rangle$ is a partial isomorphism.

Example 4 shows that, for a partial mapping $\langle p, r\rangle$ to be a partial isomorphism, $\operatorname{dom}(r)$ is not necessarily a substructure of $\mathbf{M}$ (similarly, it is easy to obtain an example showing that $\operatorname{dom}(p)$ is not necessarily a subalgebra of $\boldsymbol{A}$ ).

From Definition 2 it easily follows a particular way in which, in the case of relational languages, partial isomorphisms preserve atomic formulas:

Lemma 5. Let $\mathcal{P}$ be a relational language and $\langle p, r\rangle$ a partial isomorphism between $\mathcal{P}$-structures $\langle\boldsymbol{A}, \mathbf{M}\rangle$ and $\langle\boldsymbol{B}, \mathbf{N}\rangle$. Then, for every atomic formula $\varphi\left(x_{1}, \ldots, x_{n}\right)$, and $d_{1}, \ldots, d_{n} \in \operatorname{dom}(r)$,

$$
\left\|\varphi\left(d_{1}, \ldots, d_{n}\right)\right\|_{\mathbf{M}}^{\boldsymbol{A}} \in \mathcal{F}^{\boldsymbol{A}} \Rightarrow\left\|\varphi\left(r\left(d_{1}\right), \ldots, r\left(d_{n}\right)\right)\right\|_{\mathbf{N}}^{\boldsymbol{B}} \in \mathcal{F}^{\boldsymbol{B}} .
$$

The previous lemma cannot be extended to languages with functional symbols. Indeed, consider in Example 4 the formula $R(x \oplus y, y)$. Then it is easy to compute that $\|R(x \oplus y, y)(2,1)\|_{\mathbf{M}}^{\mathbf{A}}=1 \in \mathcal{F}^{\mathbf{A}}$ but $\|R(x \oplus y, y)(r(2), r(1))\|_{\mathbf{N}}^{\mathbf{A}}=$ $0 \notin \mathcal{F}^{\mathbf{A}}$.

We can now use partial isomorphisms to introduce back-and-forth systems between fuzzy first-order structures.

Definition 6 (Finitely isomorphic structures). Two $\mathcal{P}$-structures $\langle\boldsymbol{A}, \mathbf{M}\rangle$ and $\langle\boldsymbol{B}, \mathbf{N}\rangle$ are said to be finitely isomorphic, written $\langle\boldsymbol{A}, \mathbf{M}\rangle \cong_{f}\langle\boldsymbol{B}, \mathbf{N}\rangle$, if there is a sequence $\left\langle I_{n} \mid n \in \mathrm{N}\right\rangle$ with the following properties:

1. Every $I_{n}$ is a non-empty set of partial isomorphisms from $\langle\boldsymbol{A}, \mathbf{M}\rangle$ to $\langle\boldsymbol{B}, \mathbf{N}\rangle$.

2. For each $n \in \mathrm{N}, I_{n+1} \subseteq I_{n}$. 
3. (Forth-property I) For every $\langle p, r\rangle \in I_{n+1}$ and $m \in M$, there is $\left\langle p, r^{\prime}\right\rangle \in I_{n}$ such that $r \subseteq r^{\prime}$ and $m \in \operatorname{dom}\left(r^{\prime}\right)$.

4. (Back-property I) For every $\langle p, r\rangle \in I_{n+1}$ and $n \in N$, there is $\left\langle p, r^{\prime}\right\rangle \in I_{n}$ such that $r \subseteq r^{\prime}$ and $n \in \operatorname{rg}\left(r^{\prime}\right)$.

5. (Forth-property II) For every $\langle p, r\rangle \in I_{n+1}$ and $a \in A$, there is $\left\langle p^{\prime}, r\right\rangle \in I_{n}$ such that $p \subseteq p^{\prime}$ and $a \in \operatorname{dom}\left(p^{\prime}\right)$.

6. (Back-property II) For every $\langle p, r\rangle \in I_{n+1}$ and $b \in B$, there is $\left\langle p^{\prime}, r\right\rangle \in I_{n}$ such that $p \subseteq p^{\prime}$ and $b \in \operatorname{rg}\left(p^{\prime}\right)$.

Definition 7 (n-finitely isomorphic structures). Given a natural number n, we say that two $\mathcal{P}$-structures $\langle\boldsymbol{A}, \mathbf{M}\rangle$ and $\langle\boldsymbol{B}, \mathbf{N}\rangle$ are $n$-finitely isomorphic, written $\langle\boldsymbol{A}, \mathbf{M}\rangle \cong_{n}\langle\boldsymbol{B}, \mathbf{N}\rangle$, if there is a sequence $\left\langle I_{m} \mid m \leq n\right\rangle$ satisfying the properties of the previous definition.

\section{Elementary equivalence in fuzzy first-order models}

In this section we show that, in fuzzy first-order models, the usual classical notion of elementary equivalence can be generalized in three different meaningful ways. We will define them and give examples to show that they are not equivalent.

${ }_{220}$ Definition 8. Given two UL-chains $\boldsymbol{A}$ and $\boldsymbol{B}$, we say that two $\mathcal{P}$-models $\langle\boldsymbol{A}, \mathbf{M}\rangle$ and $\langle\boldsymbol{B}, \mathbf{N}\rangle$ are elementarily equivalent (in symbols: $\langle\boldsymbol{A}, \mathbf{M}\rangle \equiv\langle\boldsymbol{B}, \mathbf{N}\rangle$ ) if they are models of the same sentences, i.e., for every $\mathcal{P}$-sentence $\sigma,\|\sigma\|_{\mathbf{M}}^{\boldsymbol{A}} \in$ $\mathcal{F}^{A}$ if and only if $\|\sigma\|_{\mathbf{N}}^{B} \in \mathcal{F}^{B}$.

Definition 9. Given a UL-chain $\boldsymbol{A}$, we say that two $\mathcal{P}$-models $\langle\boldsymbol{A}, \mathbf{M}\rangle$ and ${ }_{225}\langle\boldsymbol{A}, \mathbf{N}\rangle$ are filter-strongly elementarily equivalent (in symbols: $\langle\boldsymbol{A}, \mathbf{M}\rangle \equiv^{\text {fs }}$ $\langle\boldsymbol{A}, \mathbf{N}\rangle)$ if they are models of the same sentences to the same degree, i.e. for every $\mathcal{P}$-sentence $\sigma,\|\sigma\|_{\mathbf{M}}^{\boldsymbol{A}} \in \mathcal{F}^{\boldsymbol{A}}$ if and only if $\|\sigma\|_{\mathbf{N}}^{\boldsymbol{A}} \in \mathcal{F}^{\boldsymbol{A}}$ and, moreover, $\|\sigma\|_{\mathbf{M}}^{\boldsymbol{A}}=\|\sigma\|_{\mathbf{N}}^{\boldsymbol{A}}$ whenever $\|\sigma\|_{\mathbf{M}}^{\boldsymbol{A}} \in \mathcal{F}^{\boldsymbol{A}}$.

These two notions are clearly equivalent for logics with weakening, because 230 then $\mathcal{F}^{\boldsymbol{A}}=\left\{\overline{1}^{\boldsymbol{A}}\right\}$. We can add yet a stronger one. 
Definition 10. Given a UL-chain $\boldsymbol{A}$, we say that two $\mathcal{P}$-models $\langle\boldsymbol{A}, \mathbf{M}\rangle$ and $\langle\boldsymbol{A}, \mathbf{N}\rangle$ are strongly elementarily equivalent (in symbols: $\langle\boldsymbol{A}, \mathbf{M}\rangle \equiv^{s}\langle\boldsymbol{A}, \mathbf{N}\rangle$ ) if for every $\mathcal{P}$-sentence $\sigma,\|\sigma\|_{\mathbf{M}}^{\boldsymbol{A}}=\|\sigma\|_{\mathbf{N}}^{\boldsymbol{A}}$.

The following lemma (a generalization of [16, Propositions 6.1 and 6.2]) will be useful to obtain examples of elementarily equivalent models.

Lemma 11. Let $\boldsymbol{A}$ and $\boldsymbol{B}$ be two UL-chains, $\langle\boldsymbol{A}, \mathbf{M}\rangle$ and $\langle\boldsymbol{B}, \mathbf{N}\rangle$ two $\mathcal{P}$-models and let $\langle f, g\rangle$ be a strong homomorphism from $\langle\boldsymbol{A}, \mathbf{M}\rangle$ to $\langle\boldsymbol{B}, \mathbf{N}\rangle$. If $f$ is a $\sigma$ mapping and $g$ is onto, then $\langle f, g\rangle$ is an elementary homomorphism. Moreover, if $f$ is one-to-one, we have $\langle\boldsymbol{A}, \mathbf{M}\rangle \equiv\langle\boldsymbol{B}, \mathbf{N}\rangle$.

Proof. Let $\langle f, g\rangle$ be a strong homomorphism such that $f$ is a $\sigma$-mapping and $g$ is an onto mapping. By induction on the complexity of the formulas we show that $\langle f, g\rangle$ is an elementary homomorphism, that is, for every formula $\varphi\left(x_{1}, \ldots, x_{n}\right)$, and $d_{1}, \ldots, d_{n} \in M$,

$$
f\left(\left\|\varphi\left(d_{1}, \ldots, d_{n}\right)\right\|_{\mathbf{M}}^{\boldsymbol{A}}\right)=\left\|\varphi\left(g\left(d_{1}\right), \ldots, g\left(d_{n}\right)\right)\right\|_{\mathbf{N}}^{\boldsymbol{B}}
$$

- Let $\varphi\left(x_{1}, \ldots, x_{n}\right)$ be atomic. Suppose that $\varphi=P\left(t_{1}, \ldots, t_{k}\right)\left(x_{1}, \ldots, x_{n}\right)$. We first prove that, for every $\mathcal{P}$-term $t\left(x_{1}, \ldots, x_{n}\right)$, and every $d_{1}, \ldots, d_{n}$,

$$
g\left(\left\|t\left(d_{1}, \ldots, d_{n}\right)\right\|_{\mathbf{M}}\right)=\left\|t\left(g\left(d_{1}\right), \ldots, g\left(d_{n}\right)\right)\right\|_{\mathbf{N}} .
$$

We proceed by induction over the complexity of the term. If $t$ is a variable $x$, then $g\left(\|x(d)\|_{\mathbf{M}}\right)=g(d)=\|x(g(d))\|_{\mathbf{N}}$. Assume that the inductive hypothesis holds for the terms $t_{1}, \ldots, t_{k}$ and let $t=F\left(t_{1}, \ldots, t_{k}\right)$, where $F$ is a $k$-ary functional symbol and the variables of the terms $t_{i}$ are in $\left\{x_{1}, \ldots, x_{n}\right\}$. We can write the following chain of equalities: 


$$
\begin{aligned}
g\left(\left\|F\left(t_{1}, \ldots, t_{k}\right)\left(d_{1}, \ldots, d_{n}\right)\right\|_{\mathbf{M}}\right) & = \\
g\left(F_{\mathbf{M}}\left(\left\|t_{1}\left(d_{1}, \ldots, d_{n}\right)\right\|_{\mathbf{M}}, \ldots,\left\|t_{k}\left(d_{1}, \ldots, d_{n}\right)\right\|_{\mathbf{M}}\right)\right) & = \\
F_{\mathbf{N}}\left(g\left(\left\|t_{1}\left(d_{1}, \ldots, d_{n}\right)\right\|_{\mathbf{M}}\right), \ldots, g\left(\left\|t_{k}\left(d_{1}, \ldots, d_{n}\right)\right\|_{\mathbf{M}}\right)\right) & = \\
F_{\mathbf{N}}\left(\left\|t_{1}\left(g\left(d_{1}\right), \ldots, g\left(d_{n}\right)\right)\right\|_{\mathbf{N}}, \ldots,\left\|t_{k}\left(g\left(d_{1}\right), \ldots, g\left(d_{n}\right)\right)\right\|_{\mathbf{N}}\right) & = \\
\left\|F\left(t_{1}, \ldots, t_{k}\right)\left(g\left(d_{1}\right), \ldots, g\left(d_{n}\right)\right)\right\|_{\mathbf{N}} &
\end{aligned}
$$
algebras and the third equality is justified by applying the inductive hypothesis.

Now given the atomic formula $P\left(t_{1}, \ldots, t_{k}\right)\left(x_{1}, \ldots, x_{n}\right)$, we have:

$$
\begin{array}{r}
f\left(\left\|P\left(t_{1}, \ldots, t_{k}\right)\left(d_{1}, \ldots, d_{n}\right)\right\|_{\mathbf{M}}^{\mathbf{A}}\right)= \\
f\left(P_{\mathbf{M}}\left(\left\|t_{1}\left(d_{1}, \ldots, d_{n}\right)\right\|_{\mathbf{M}}, \ldots,\left\|t_{k}\left(d_{1}, \ldots, d_{n}\right)\right\|_{\mathbf{M}}\right)\right)= \\
P_{\mathbf{N}}\left(g\left(\left\|t_{1}\left(d_{1}, \ldots, d_{n}\right)\right\|_{\mathbf{M}}\right), \ldots, g\left(\left\|t_{k}\left(d_{1}, \ldots, d_{n}\right)\right\|_{\mathbf{M}}\right)\right)= \\
P_{\mathbf{N}}\left(\left\|t_{1}\left(g\left(d_{1}\right), \ldots, g\left(d_{n}\right)\right)\right\|_{\mathbf{N}}, \ldots,\left\|t_{k}\left(g\left(d_{1}\right), \ldots, g\left(d_{n}\right)\right)\right\|_{\mathbf{N}}\right)= \\
\left\|P\left(t_{1}, \ldots, t_{k}\right)\left(g\left(d_{1}\right), \ldots, g\left(d_{n}\right)\right)\right\|_{\mathbf{N}} .
\end{array}
$$

The second equality is due to the fact that $\langle f, g\rangle$ is a strong homomorphism and the third equality is justified by applying (1).

- Now, let $\lambda \in \mathcal{L}$ be a $k$-ary connective, and

$$
\varphi\left(x_{1}, \ldots, x_{n}\right)=\lambda\left(\psi_{1}, \ldots, \psi_{k}\right)\left(x_{1}, \ldots, x_{n}\right) .
$$

Assume inductively that the property holds for the formulas $\psi_{1}, \ldots, \psi_{k}$. We have: 


$$
\begin{array}{r}
f\left(\left\|\lambda\left(\psi_{1}, \ldots, \psi_{k}\right)\left(d_{1}, \ldots, d_{n}\right)\right\|_{\mathbf{M}}^{\boldsymbol{A}}\right)= \\
f\left(\lambda^{\boldsymbol{A}}\left(\left\|\psi_{1}\left(d_{1}, \ldots, d_{n}\right)\right\|_{\mathbf{M}}^{\boldsymbol{A}}, \ldots,\left\|\psi_{k}\left(d_{1}, \ldots, d_{n}\right)\right\|_{\mathbf{M}}^{\boldsymbol{A}}\right)\right)= \\
\lambda^{\boldsymbol{B}}\left(f\left(\left\|\psi_{1}\left(d_{1}, \ldots, d_{n}\right)\right\|_{\mathbf{M}}^{\boldsymbol{A}}\right), \ldots, f\left(\left\|\psi_{k}\left(d_{1}, \ldots, d_{n}\right)\right\|_{\mathbf{M}}^{\boldsymbol{A}}\right)\right)= \\
\lambda^{\boldsymbol{B}}\left(\left\|\psi_{1}\left(g\left(d_{1}\right), \ldots, g\left(d_{n}\right)\right)\right\|_{\mathbf{N}}^{\boldsymbol{B}}, \ldots,\left\|\psi_{1}\left(g\left(d_{1}\right), \ldots, g\left(d_{n}\right)\right)\right\|_{\mathbf{N}}^{\boldsymbol{B}}\right)= \\
\left\|\lambda\left(\psi_{1}, \ldots, \psi_{k}\right)\left(g\left(d_{1}\right), \ldots, g\left(d_{n}\right)\right)\right\|_{\mathbf{N}}^{\boldsymbol{B}} .
\end{array}
$$

Moreover, if $f$ is one-to-one, we can guarantee that for every $a \in A, a \in \mathcal{F}^{\boldsymbol{A}}$ if and only if $f(a) \in \mathcal{F}^{\boldsymbol{B}}$. Indeed, if $a \geq \overline{1}^{\boldsymbol{A}}$, then $f(a) \geq f\left(\overline{1}^{\boldsymbol{A}}\right)=\overline{1}^{\boldsymbol{B}}$ (using that $f$ is a homomorphism of UL-algebras); conversely if $f(a) \geq \overline{1}^{\boldsymbol{B}}=f\left(\overline{1}^{\boldsymbol{A}}\right)$, then $f(a) \wedge f\left(\overline{1}^{\boldsymbol{A}}\right)=f\left(a \wedge \overline{1}^{\boldsymbol{A}}\right)=f\left(\overline{1}^{\boldsymbol{A}}\right)$, so by injectivity, $a \wedge \overline{1}^{\boldsymbol{A}}=\overline{1}^{\boldsymbol{A}}$. Consequently, since $\langle f, g\rangle$ is an elementary homomorphism, $\langle\boldsymbol{A}, \mathbf{M}\rangle \equiv\langle\boldsymbol{B}, \mathbf{N}\rangle$.

Similarly, observe that if there is an elementary homomorphism $\left\langle I d_{\boldsymbol{A}}, g\right\rangle$ from 
${ }_{275}\langle\boldsymbol{A}, \mathbf{M}\rangle$ to $\langle\boldsymbol{A}, \mathbf{N}\rangle$, then $\langle\boldsymbol{A}, \mathbf{M}\rangle \equiv^{s}\langle\boldsymbol{A}, \mathbf{N}\rangle$. Therefore, in all the LöwenheimSkolem theorems proved in [15] for structures over a fixed UL-chain one always obtains a (bigger or smaller) model which is strongly elementarily equivalent to the initial one.

The following example shows two models (for a logic with weakening) which are filter-strongly elementarily equivalent but not strongly elementarily equivalent.

Example 12. Consider a predicate language with only one monadic predicate $P$ and take two models over the standard Gödel chain, $\left\langle[0,1]_{\mathrm{G}}, \mathbf{M}\right\rangle$ and $\left\langle[0,1]_{\mathrm{G}}, \mathbf{N}\right\rangle$. The domain in both cases is the set of all natural numbers $\mathrm{N}$ and the interpretation of the predicate is respectively defined as:

$$
\begin{aligned}
& P_{\mathbf{M}}(n)= \begin{cases}\frac{3}{4}-\frac{1}{n}, & \text { if } n \geq 2, \\
0, & 0 \leq n \leq 1 .\end{cases} \\
& P_{\mathbf{N}}(n)= \begin{cases}\frac{1}{2}-\frac{1}{n}, & \text { if } n \geq 2, \\
0, & 0 \leq n \leq 1 .\end{cases}
\end{aligned}
$$

On the one hand, $\|(\exists x) P(x)\|_{\mathbf{M}}=\frac{3}{4}$ but $\|(\exists x) P(x)\|_{\mathbf{N}}=\frac{1}{2}$, so the models are not strongly elementarily equivalent. On the other hand, we will see that elementary equivalence still holds. Take $f$ as any non-decreasing bijection from $[0,1]$ to $[0,1]$ such that $f\left(\frac{3}{4}\right)=\frac{1}{2}, f(1)=1, f(0)=0$, and for every $n \in \mathrm{N}$, $290 f\left(\frac{3}{4}-\frac{1}{n}\right)=\frac{1}{2}-\frac{1}{n}$. It is clear that $f$ is a $\mathrm{G}$-homomorphism preserving suprema and infima. Then we can consider the $\sigma$-mapping $\langle f, I d\rangle$ and apply Lemma 11 to obtain that $\left\langle[0,1]_{\mathrm{G}}, \mathbf{M}\right\rangle \equiv\left\langle[0,1]_{\mathrm{G}}, \mathbf{N}\right\rangle$.

Now the following example shows two models which are elementarily equivalent but not filter-strongly elementarily equivalent.

Example 13. Consider the same predicate language of Example 12 and take again two models $\langle\boldsymbol{A}, \mathbf{M}\rangle$ and $\langle\boldsymbol{A}, \mathbf{N}\rangle$ with both domains $M$ and $N$ equal to the set of all natural numbers $\mathrm{N}$. We take both models over the same algebra, 
namely, the residuated lattice defined in the real unit interval by the uninorm:

$$
x \&^{\boldsymbol{A}} y= \begin{cases}\min \{x, y\}, & \text { if } y \leq 1-x, \\ \max \{x, y\}, & \text { if } y>1-x,\end{cases}
$$

and its residuum:

$$
x \rightarrow^{\boldsymbol{A}} y= \begin{cases}\max \{1-x, y\}, & \text { if } x \leq y, \\ \min \{1-x, y\}, & \text { if } y>x\end{cases}
$$

The interpretation of the unique predicate in both models is as follows:

$$
\begin{aligned}
& P_{\mathbf{M}}(n)= \begin{cases}\frac{4}{5}-\frac{1}{n^{4}}, & \text { if } n \geq 2, \\
0, & \text { if } 0 \leq n \leq 1 .\end{cases} \\
& P_{\mathbf{N}}(n)= \begin{cases}\frac{3}{5}-\frac{1}{n^{4}}, & \text { if } n \geq 2, \\
0, & \text { if } 0 \leq n \leq 1 .\end{cases}
\end{aligned}
$$

The neutral element of the uninorm is $\frac{1}{2}$. The filter is $\mathcal{F}^{\boldsymbol{A}}=\left[\frac{1}{2}, 1\right]$. Now, observe that we have: $\|(\exists x) P(x)\|_{\mathbf{M}}=\frac{4}{5}$ but $\|(\exists x) P(x)\|_{\mathbf{N}}=\frac{3}{5}$, so the models are not filter-strong elementarily equivalent because they assign different true values to the sentence $(\exists x) P(x)$. However, we can prove that they are elementarily equivalent. Indeed, consider the function $f:[0,1] \longrightarrow[0,1]$ defined as $f(x)=x$ for each $x \in\left(\frac{4}{5}, 1\right], f\left(\frac{4}{5}\right)=\frac{3}{5}$, and by taking for each interval $\left[\frac{4}{5}-\frac{1}{n^{4}}, \frac{4}{5}-\frac{1}{(n+1)^{4}}\right]$ the usual affine transformation into the interval $\left[\frac{3}{5}-\frac{1}{n^{4}}, \frac{3}{5}-\frac{1}{(n+1)^{4}}\right]$, the usual affine transformation from $\left[\frac{1}{2}, \frac{4}{5}-\frac{1}{2^{4}}\right]$ into $\left[\frac{1}{2}, \frac{3}{5}-\frac{1}{2^{4}}\right]$, and finally defining $f(x)=1-f(1-x)$ for each $\left[0, \frac{1}{2}\right)$. It is clear that $f$ preserves order, suprema and infima, it is injective and, for each $x \in[0,1], f(1-x)=1-f(x)$; using this it is easy to check that $f$ is an $\boldsymbol{A}$-homomorphism. Therefore, we can apply Lemma 11 to the elementary homomorphism $\left\langle f, I d_{\mathbf{N}}\right\rangle$ and obtain that $\langle\boldsymbol{A}, \mathbf{M}\rangle \equiv\langle\boldsymbol{A}, \mathbf{N}\rangle$.

\section{A sufficient condition for elementary equivalence}

The goal of this section is to use the characterization of elementary equivalence for classical many-sorted structures to obtain a sufficient condition for 

we have that $\langle\boldsymbol{A}, \mathbf{M}, a\rangle_{a \in A} \equiv\langle\boldsymbol{A}, \mathbf{N}, a\rangle_{a \in A}$ and thus $\langle\boldsymbol{A}, \mathbf{M}\rangle \equiv^{s}\langle\boldsymbol{A}, \mathbf{N}\rangle$.

The converse implication of the previous theorem is not true in general. Consider the following counterexample:

Example 15. Let $\mathcal{P}$ be a finite predicate language, $\mathbf{M}$ a classical one-sorted 335 finite model and $\boldsymbol{B}_{2}$ the two-element Boolean algebra. Now take an infinite Lalgebra $\boldsymbol{A}$. Since $\boldsymbol{B}_{2}$ is a subalgebra of $\boldsymbol{A}$, we can also see the classical model $\left\langle\boldsymbol{B}_{2}, \mathbf{M}\right\rangle$ as a model over the algebra $\boldsymbol{A}$, namely $\langle\boldsymbol{A}, \mathbf{M}\rangle$. Clearly then $\left\langle\boldsymbol{B}_{2}, \mathbf{M}\right\rangle \equiv$ $\langle\boldsymbol{A}, \mathbf{M}\rangle$ but it is not true that $\left\langle\boldsymbol{B}_{2}, \mathbf{M}\right\rangle \cong_{f}\langle\boldsymbol{A}, \mathbf{M}\rangle$. Otherwise the two models 
will be elementarily equivalent from the point of view of classical logic and also their algebras. This is impossible because, by the classical results, one algebra which is finite cannot be elementarily equivalent to an infinite algebra.

The following examples show how one can apply in particular cases (fuzzy models over the Łukasiewicz, Gödel and arbitrary standard UL-algebras) the condition of Theorem 14 to obtain elementary equivalence.

Example 16. Let $\mathcal{P}$ be a predicate language with a unique binary relational symbol E. Consider the $\mathcal{P}$-models $\left\langle[0,1]_{€}, \mathbf{N}_{1}\right\rangle$ and $\left\langle[0,1]_{€}, \mathbf{N}_{2}\right\rangle$, where the domains of $\mathbf{N}_{1}$ and $\mathbf{N}_{2}$ are $[0,1]^{\mathbf{Q}}$ and $[0,1]$, the rational and the real unit interval respectively. The relations $E^{\mathbf{N}_{1}}$ and $E^{\mathbf{N}_{2}}$ (similarity relations) are defined as follows:

$$
\begin{gathered}
E^{\mathbf{N}_{1}}(x, y)=1-|x-y|, \text { for every } x, y \in[0,1]^{\mathrm{Q}}, \\
E^{\mathbf{N}_{2}}(x, y)=1-|x-y|, \text { for every } x, y \in[0,1]
\end{gathered}
$$

Now we show that $\left\langle[0,1]_{\mathrm{E}}, \mathbf{N}_{1}\right\rangle \equiv\left\langle[0,1]_{\mathrm{E}}, \mathbf{N}_{2}\right\rangle$ by building a system of partial isomorphisms and using Theorem 14. Observe that a partial isomorphism between $\left\langle[0,1]_{\mathrm{E}}, \mathbf{N}_{1}\right\rangle$ and $\left\langle[0,1]_{\mathrm{E}}, \mathbf{N}_{2}\right\rangle$ is given by the set of pairs $\left\langle\operatorname{Id}_{[0,1]}, r\right\rangle$, where $r$ is a bijection, $\operatorname{dom}(r) \subseteq[0,1]^{\mathrm{Q}}$, and $\operatorname{rg}(r) \subseteq[0,1]$ in such a way that, for every $x, y \in \operatorname{dom}(r),|x-y|=|f(x)-f(y)|$. Then, we define a system of partial isomorphisms where, for each $n \in \mathbb{N}, I_{n}$ is the set of all the finite partial isomorphisms with the identity for the algebraic part. Now we prove that conditions $1-6$ in Definition 6 are satisfied. Condition 1: Observe that $\left\langle\operatorname{Id}_{[0,1]}, \emptyset\right\rangle \in I_{n}$; therefore, $I_{n} \neq \emptyset$. Condition 2 is obvious. Condition 3: Suppose that $\langle I d, r\rangle \in I_{n+1}$ and let $d \in[0,1]^{\mathrm{Q}}$. Suppose also that dom $=\left\{d_{1}, \ldots, d_{k}\right\}$. Take an element $355 e \in[0,1]$ such that $\left|d_{1}-d\right|=\left|r\left(d_{1}\right)-e\right|$ and take $r^{\prime}=r \cup\{\langle d, e\rangle\}$. Condition 4 is analogously proved and conditions 5 and 6 are trivial.

Example 17. Let us consider the predicate language $\mathcal{P}$ in Example 16 , and the $\mathcal{P}$-models $\left\langle[0,1]_{\mathrm{G}}, \mathbf{M}_{1}\right\rangle$ and $\left\langle[0,1]_{\mathrm{G}}^{\mathrm{Q}}, \mathbf{M}_{2}\right\rangle$, where $[0,1]_{\mathrm{G}}^{\mathrm{Q}}$ is the restriction of the algebra $[0,1]_{\mathrm{G}}$ to the rational numbers, and where the domains of $\mathbf{M}_{1}$ and $\mathbf{M}_{2}$ are $[0,1]$ and $[0,1]^{\mathbf{Q}}$, respectively. The relations $E^{\mathbf{M}_{1}}$ and $E^{\mathbf{M}_{2}}$ are both defined 
in the same form as follows:

$$
E^{\mathbf{M}_{i}}(x, y)= \begin{cases}1, & \text { if } x=y, \\ \min \{x, y\}, & \text { otherwise. }\end{cases}
$$

When $x=y$, we have:

$$
p\left(E^{\mathbf{M}_{1}}(x, y)\right)=E^{\mathbf{M}_{1}}(r(x), r(y)) .
$$

If $x \neq y$, then we have: $p(\min \{x, y\})=\min \{r(x), r(y)\}$.

We define a a system of partial isomorphisms where, for each $n \in \mathrm{N}, I_{n}$ is the set of all the pairs $\langle p, r\rangle$ such that $p$ and $r$ coincide over $\operatorname{dom}(p) \cup \operatorname{dom}(r)$ and they are finite partial isomorphisms preserving the order.

Example 18. Let $\mathcal{P}$ be a predicate language with a unique binary relational symbol $E$ and let $\boldsymbol{A}$ be a standard UL-chain. Consider a $\mathcal{P}$-model $\langle\boldsymbol{A}, \mathbf{M}\rangle$ with the following properties:

1. $E^{\mathrm{M}}$ is a similarity.

2. For each $a \in[0,1]$, there are $d, e \in M$ such that $E^{\mathbf{M}}(d, e)=a$.

3. For every $d \in M$, the set $[d]=\left\{e \in M \mid E^{\mathbf{M}}(d, e)>0\right\}$ is infinite.

4. For every $d \in M$, the set $M \backslash[d]$ is also infinite.

5. For every $d, e \in M$, if $e \notin[d],[d] \cap[e]=\emptyset$.

6. For every $d \in M$ there is a unique $a \in A, 0<a<1$ such that for every $e \in[d], e \neq d, E^{\mathbf{M}}(d, e)=a$.

Notice that, if we define for each $a \in(0,1)$ the set $M_{a}=\{d \in M \mid$ there is $e \in M$ with $\left.E^{\mathbf{M}}(d, e)=a\right\}$, then $\left\{M_{a} \mid a \in(0,1)\right\}$ is a partition of the domain $M$.

Take another model $\langle\boldsymbol{A}, \mathbf{N}\rangle$ with analogous properties on a possibly different domain. Let $\langle p, r\rangle$ be a partial mapping from $\langle\boldsymbol{A}, \mathbf{M}\rangle$ to $\langle\boldsymbol{A}, \mathbf{N}\rangle$ such that:

- $p$ is the identity on A,

- $r$ is injective, 
- for every $d \in \operatorname{dom}(r)$, if $d \in M_{a}$ then $r(d) \in N_{a}$.

Then $\langle p, r\rangle$ is a partial isomorphism. We can define a system of partial isomorphisms where, for each $n \in \mathbb{N}, I_{n}$ is the set of all such finite partial isomorphisms. Notice that the sets $M_{a}$ and $N_{a}$ may have different cardinalities.

\section{Finitely isomorphic fuzzy first-order models}

By using the notion of $n$-finitely isomorphic structures introduced in Definition 7 , in this section we aim at refining the results of the previous one in such a way that back-and-forth systems only use as many layers as necessary to guarantee that two models are $n$-elementarily equivalent (that is to say, in order to be models of the same sentences up until a given complexity $n$ ). The complexity of the sentences is measured using a modification of the syntactic degree of formulas introduced by Hájek in [22, Definition 5.6.7].

The results in this section are achieved in a purely non-classical approach In this case, the translation to two-sorted structures and classical results is not useful because it would not be so finely grained. The reason is that, intuitively speaking, the translation does not preserve the rank of the formulas involved, making it, in some cases, actually much bigger.

Definition 19 (Nested rank of a formula). Let $\mathcal{P}$ be a predicate language. Given a $\mathcal{P}$-formula $\varphi$ we define by induction the nested rank of $\varphi$, denoted by $N R(\varphi)$, as follows.

- If $\varphi$ is atomic (given by a predicate or a 0-ary connective), $N R(\varphi)=0$.

- For every $n \geq 1$, every $\mathcal{P}$-formulas $\varphi_{1}, \ldots, \varphi_{n}$ and every $n$-ary connective $\lambda \in \mathcal{L}$,

$$
N R\left(\lambda\left(\varphi_{1}, \ldots, \varphi_{n}\right)=N R\left(\varphi_{1}\right)+\ldots+N R\left(\varphi_{n}\right)+1\right.
$$

- For every $\mathcal{P}$-formula $\varphi, N R((\forall x) \varphi)=N R((\exists x) \varphi)=N R(\varphi)+1$.

Observe that, by the definition of nested rank, if both $\mathcal{L}$ and $\mathcal{P}$ are finite, and we fix a finite set of variables $V_{0}$, then, for any $n \geq 0$, the set $\{\varphi \mathcal{P}$-formula in variables in $\left.V_{0} \mid N R(\varphi) \leq n\right\}$ is finite. 
Definition 20. Given $\mathcal{P}$-models $\langle\boldsymbol{A}, \mathbf{M}\rangle$ and $\langle\boldsymbol{B}, \mathbf{N}\rangle$, we write

$$
\langle\boldsymbol{A}, \mathbf{M}\rangle \equiv_{n}\langle\boldsymbol{B}, \mathbf{N}\rangle
$$

whenever $\langle\boldsymbol{A}, \mathbf{M}\rangle$ and $\langle\boldsymbol{B}, \mathbf{N}\rangle$ are models of the same sentences of nested rank $\leq n$.

Analogously, we can define strong $n$-equivalence from models over the same algebra:

Definition 21. Given two $\mathcal{P}$-models $\langle\boldsymbol{A}, \mathbf{M}\rangle$ and $\langle\boldsymbol{A}, \mathbf{N}\rangle$, we write

$$
\langle\boldsymbol{A}, \mathbf{M}\rangle \equiv_{n}^{s}\langle\boldsymbol{A}, \mathbf{N}\rangle
$$

whenever $\|\sigma\|_{\mathbf{M}}^{\boldsymbol{A}}=\|\sigma\|_{\mathbf{N}}^{\boldsymbol{A}}$, for every $\mathcal{P}$-sentence $\sigma$ of nested rank $\leq n$.

We are ready to present the non-classical proof of Theorem 14 for the case of strong $n$-equivalence using, without loss of generality, only finite relational languages.

Theorem 22. Let $\mathcal{P}$ be a finite relational predicate language, $\langle\boldsymbol{A}, \mathbf{M}\rangle$ and $\langle\boldsymbol{A}, \mathbf{N}\rangle$ be $\mathcal{P}$-models and $n \in \mathbf{N}$. Assume that $\langle\boldsymbol{A}, \mathbf{M}\rangle \cong_{n}\langle\boldsymbol{A}, \mathbf{N}\rangle$ via a system $\left\langle I_{m} \mid m \leq n\right\rangle$ such that for every $m \leq n$, and every $\langle p, r\rangle \in I_{m}, p\left\lceil A \subseteq I d_{A}\right.$. Then $\langle\boldsymbol{A}, \mathbf{M}\rangle \equiv_{n}^{s}\langle\boldsymbol{A}, \mathbf{N}\rangle$.

(*) For every formula $\varphi\left(x_{1}, \ldots, x_{k}\right),\langle p, r\rangle \in I_{m}$ with $N R(\varphi) \leq m \leq n$, and $d_{1}, \ldots, d_{k} \in \operatorname{dom}(r),\left\|\varphi\left(d_{1}, \ldots, d_{k}\right)\right\|_{\mathbf{M}}^{\boldsymbol{A}}=\left\|\varphi\left(r\left(d_{1}\right), \ldots, r\left(d_{k}\right)\right)\right\|_{\mathbf{N}}^{\boldsymbol{A}}$.

- Let $R\left(x_{1}, \ldots, x_{k}\right)$ be an atomic formula. Since $\langle p, r\rangle$ is a partial isomorphism, by condition 5 of Definition 2 we have:

$$
R_{\mathbf{M}}\left(d_{1}, \ldots, d_{k}\right)=R_{\mathbf{N}}\left(r\left(d_{1}\right), \ldots, r\left(d_{k}\right)\right) .
$$

- Consider now the formula $\lambda\left(\alpha_{1}, \ldots, \alpha_{l}\right)$ in variables $\left\{x_{1}, \ldots, x_{k}\right\}$, where $\lambda$ is an $l$-ary connective with $l \geq 1$. Let $\langle p, r\rangle \in I_{m}$ with $N R\left(\lambda\left(\alpha_{1}, \ldots, \alpha_{l}\right)\right) \leq m \leq n$, 
and $d_{1}, \ldots, d_{k} \in \operatorname{dom}(r)$. Assume inductively that the property $(*)$ holds for $\alpha_{1}, \ldots, \alpha_{l}$. By Definition 19 we have:

$$
N R\left(\lambda\left(\alpha_{1}, \ldots, \alpha_{l}\right)\right)=N R\left(\alpha_{1}\right)+\ldots+N R\left(\alpha_{l}\right)+1
$$

then $N R\left(\alpha_{i}\right) \leq m-1$ for each $i \in\{1, \ldots, l\}$. By Definition 6 we have that $\langle p, r\rangle \in I_{m-1}$. Then we apply the inductive hypothesis and we obtain for each $i \in\{1, \ldots, l\}$,

$$
\left\|\alpha_{i}\left(d_{1}, \ldots, d_{k}\right)\right\|_{\mathbf{M}}^{\boldsymbol{A}}=\left\|\alpha_{i}\left(r\left(d_{1}\right), \ldots, r\left(d_{k}\right)\right)\right\|_{\mathbf{N}}^{\boldsymbol{A}} .
$$

Therefore,

$$
\left\|\lambda\left(\alpha_{1}, \ldots, \alpha_{l}\right)\left[d_{1}, \ldots, d_{k}\right]\right\|_{\mathbf{M}}^{\boldsymbol{A}}=\left\|\lambda\left(\alpha_{1}, \ldots, \alpha_{l}\right)\left[r\left(d_{1}\right), \ldots, r\left(d_{k}\right)\right]\right\|_{\mathbf{N}}^{\boldsymbol{A}}
$$

- Let now $(\exists y) \varphi\left(y, x_{1}, \ldots, x_{k}\right)$ be an existential formula, $\langle p, r\rangle \in I_{m}$ with $N R((\exists y) \varphi) \leq m \leq n$, and $d_{1}, \ldots, d_{k} \in \operatorname{dom}(r)$. By Definition 19 we have that $N R((\exists y) \varphi)=N R(\varphi)+1$ and thus $N R(\varphi) \leq m-1$. For every $d \in M$, by the (Forth Property I) of Definition 6, there is $\left\langle p, r^{\prime}\right\rangle \in I_{m-1}$ such that $r \subseteq r^{\prime}$ and $d \in \operatorname{dom}\left(r^{\prime}\right)$. Then, by the inductive hypothesis,

$$
\left\|\varphi\left(d, d_{1}, \ldots, d_{k}\right)\right\|_{\mathbf{M}}^{\boldsymbol{A}}=\left\|\varphi\left(r^{\prime}(d), r^{\prime}\left(d_{1}\right), \ldots, r^{\prime}\left(d_{k}\right)\right)\right\|_{\mathbf{N}}^{\boldsymbol{A}} .
$$

The same argument can be done for every $e \in N$ using the (Back Property I) of Definition 6. Consequently, since $r \subseteq r^{\prime}$ and $d_{1}, \ldots, d_{k} \in \operatorname{dom}(r)$,

$$
\left\{\left\|\varphi\left(d, d_{1}, \ldots, d_{k}\right)\right\|_{\mathbf{M}}^{\boldsymbol{A}} \mid d \in M\right\}=\left\{\| \varphi\left(e, r\left(d_{1}\right), \ldots, r\left(d_{k}\right) \|_{\mathbf{N}}^{\boldsymbol{A}} \mid e \in N\right\} .\right.
$$

We can conclude that $\left\|(\exists y) \varphi\left(d_{1}, \ldots, d_{k}\right)\right\|_{\mathbf{M}}^{\boldsymbol{A}}=\left\|(\exists y) \varphi\left(r\left(d_{1}\right), \ldots, r\left(d_{k}\right)\right)\right\|_{\mathbf{N}}^{\boldsymbol{A}}$. The case of the universal formulas is anologous. Finally, from $(*)$ if we consider only sentences $\varphi$, it follows that $\langle\boldsymbol{A}, \mathbf{M}\rangle \equiv_{n}^{s}\langle\boldsymbol{A}, \mathbf{N}\rangle$.

We now present a counterexample to show that the right-to-left implication does not always hold.

Example 23. Consider a predicate language with only one monadic predicate symbol $P$ and take two models over any $[0,1]$-valued chain $\boldsymbol{A},\langle\boldsymbol{A}, \mathbf{M}\rangle$ and 
$\langle\boldsymbol{A}, \mathbf{N}\rangle$. The domain of $\mathbf{M}$ is the set of all natural numbers $\mathbf{N}$ and the domain of $\mathbf{N}$ is $\mathbf{N} \cup\{\infty\}$ (the natural numbers enriched with a new element). The interpretations of the predicate are respectively defined as:

$$
\begin{gathered}
P_{\mathbf{M}}(n)= \begin{cases}1-\frac{1}{n}, & \text { if } n \geq 1, \\
0, & \text { if } n=0 .\end{cases} \\
P_{\mathbf{N}}(n)= \begin{cases}1-\frac{1}{n}, & \text { if } n \in \mathrm{N}, \text { and } n \geq 1, \\
0, & \text { if } n=0, \\
1, & \text { if } n=\infty .\end{cases}
\end{gathered}
$$

Observe that the only sentences of nested rank 0 or 1 are 0-ary connectives or sentences of the form $(\forall x) P(x)$ or $(\exists x) P(x)$, and thus clearly $\langle\boldsymbol{A}, \mathbf{M}\rangle \equiv_{1}^{s}$ $\langle\boldsymbol{A}, \mathbf{N}\rangle$. However, it is not the case that $\langle\boldsymbol{A}, \mathbf{M}\rangle \sim_{1}\langle\boldsymbol{A}, \mathbf{N}\rangle$, because in the domain of $\mathbf{M}$ no element could be related to $\infty$ in any partial isomorphism, due to condition 4 of Definition 2).

Finally, we obtain another non-classical proof of Theorem 14 for the case of elementary equivalence when the two models have different algebras. For the proof of the following theorem we will slightly modify the definition of rank of the existential and universal formulas as follows:

$$
N R((\exists y) \varphi)=N R((\forall y) \varphi)=N R(\varphi)+3
$$

Theorem 24. Let $\mathcal{P}$ be a finite relational predicate language and $\langle\boldsymbol{A}, \mathbf{M}\rangle$ and $\langle\boldsymbol{B}, \mathbf{N}\rangle$ be $\mathcal{P}$-models. The following holds for every $n \in \mathbf{N}$ :

$$
\langle\boldsymbol{A}, \mathbf{M}\rangle \cong_{n}\langle\boldsymbol{B}, \mathbf{N}\rangle \quad \Rightarrow \quad\langle\boldsymbol{A}, \mathbf{M}\rangle \equiv_{n}\langle\boldsymbol{B}, \mathbf{N}\rangle
$$

Proof. Assume that $\langle\boldsymbol{A}, \mathbf{M}\rangle \cong_{n}\langle\boldsymbol{B}, \mathbf{N}\rangle$ via $\left\langle I_{m} \mid m \leq n\right\rangle$. By induction on the complexity of the formulas we show that

(**) For every formula $\varphi\left(x_{1}, \ldots, x_{k}\right),\langle p, r\rangle \in I_{m}$ with $N R(\varphi) \leq m \leq n$, and $d_{1}, \ldots, d_{k} \in \operatorname{dom}(r)$, there is a pair $\left\langle p^{\prime}, r^{\prime}\right\rangle \in I_{m-N R(\varphi)}$ with $p \subseteq p^{\prime}, r \subseteq r^{\prime}$, such that $\left\|\varphi\left(d_{1}, \ldots, d_{k}\right)\right\|_{\mathbf{M}}^{\boldsymbol{A}} \in \operatorname{dom}\left(p^{\prime}\right)$, and

$$
p^{\prime}\left(\left\|\varphi\left(d_{1}, \ldots, d_{k}\right)\right\|_{\mathbf{M}}^{\boldsymbol{A}}\right)=\left\|\varphi\left(r^{\prime}\left(d_{1}\right), \ldots, r^{\prime}\left(d_{k}\right)\right)\right\|_{\mathbf{N}}^{\boldsymbol{B}}
$$


- Let $R\left(x_{1}, \ldots, x_{k}\right)$ be an atomic formula. Since $\langle p, r\rangle$ is a partial isomorphism, by condition 4 of Definition 2 we have:

$$
p\left(R_{\mathbf{M}}\left(d_{1}, \ldots, d_{k}\right)\right)=R_{\mathbf{N}}\left(r\left(d_{1}\right), \ldots, r\left(d_{k}\right)\right) .
$$

- Consider now the formula $\lambda\left(\alpha_{1}, \ldots, \alpha_{l}\right)$ in the variables $\left\{x_{1}, \ldots, x_{k}\right\}$, where $\lambda$ is an $l$-ary connective with $l \geq 1$. Let $\langle p, r\rangle \in I_{m}$ with $N R\left(\lambda\left(\alpha_{1}, \ldots, \alpha_{l}\right)\right) \leq$ $m \leq n$, and $d_{1}, \ldots, d_{k} \in \operatorname{dom}(r)$. Assume inductively that the property $(* *)$ holds for $\alpha_{1}, \ldots, \alpha_{l}$. By Definition 19 we have:

$$
N R\left(\lambda\left(\alpha_{1}, \ldots, \alpha_{l}\right)\right)=N R\left(\alpha_{1}\right)+\ldots+N R\left(\alpha_{l}\right)+1
$$

We define now two sequences of partial mappings:

$$
p \subseteq p_{1} \subseteq p_{2} \subseteq \ldots \subseteq p_{l},
$$

and

$$
r \subseteq r_{1} \subseteq r_{2} \subseteq \ldots \subseteq r_{l}
$$

such that

i) $\left\|\alpha_{j}\left(d_{1}, \ldots, d_{k}\right)\right\|_{\mathbf{M}}^{\boldsymbol{A}} \in \operatorname{dom}\left(p_{i}\right)$, for $j \leq i \leq l$,

ii) $\left\langle p_{i}, r_{i}\right\rangle \in I_{m-\left(N R\left(\alpha_{1}\right)+\ldots+N R\left(\alpha_{i}\right)\right)}$,

iii) $p_{i}\left(\left\|\alpha_{j}\left(d_{1}, \ldots, d_{k}\right)\right\|_{\mathbf{M}}^{\boldsymbol{A}}\right)=\left\|\alpha_{j}\left(r_{i}\left(d_{1}\right), \ldots, r_{i}\left(d_{k}\right)\right)\right\|_{\mathbf{N}}^{B}$, for $j \leq i \leq l$.

By inductive hypothesis, there is $\left\langle p_{1}, r_{1}\right\rangle \in I_{m-N R\left(\alpha_{1}\right)}$ with $p \subseteq p_{1}, r \subseteq r_{1}$ such that $\left\|\alpha_{1}\left(d_{1}, \ldots, d_{k}\right)\right\|_{\mathbf{M}}^{\boldsymbol{A}} \in \operatorname{dom}\left(p_{1}\right)$, and

$$
p_{1}\left(\left\|\alpha_{1}\left(d_{1}, \ldots, d_{k}\right)\right\|_{\mathbf{M}}^{\boldsymbol{A}}\right)=\left\|\alpha_{1}\left(r_{1}\left(d_{1}\right), \ldots, r_{1}\left(d_{k}\right)\right)\right\|_{\mathbf{N}}^{\boldsymbol{B}} .
$$

By iterating this same procedure we can obtain the desired sequences.

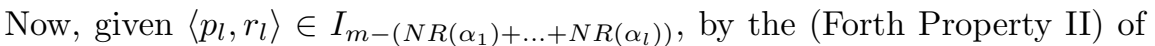

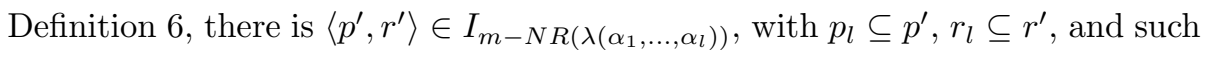
that $\left\|\lambda\left(\alpha_{1}, \ldots, \alpha_{l}\right)\left(d_{1}, \ldots, d_{k}\right)\right\|_{\mathbf{M}}^{\boldsymbol{A}} \in \operatorname{dom}\left(p^{\prime}\right)$. Finally, by Definition 2 ,

$$
p^{\prime}\left(\left\|\lambda\left(\alpha_{1}, \ldots, \alpha_{l}\right)\left(d_{1}, \ldots, d_{k}\right)\right\|_{\mathbf{M}}^{\boldsymbol{A}}\right)=\left\|\lambda\left(\alpha_{1}, \ldots, \alpha_{l}\right)\left(r^{\prime}\left(d_{1}\right), \ldots, r^{\prime}\left(d_{k}\right)\right)\right\|_{\mathbf{N}}^{B} .
$$


- Let $(\exists y) \varphi\left(y, x_{1}, \ldots, x_{k}\right)$ be an existential formula, $\langle p, r\rangle \in I_{m}$ with $N R((\exists y) \varphi) \leq$ $m \leq n$, and $d_{1}, \ldots, d_{k} \in \operatorname{dom}(r)$. By the modified definition of rank we have stated just before the statement of this theorem, we have $N R((\exists y) \varphi)=$ $N R(\varphi)+3$. Now we show that there is $\left\langle p^{\prime}, r^{\prime}\right\rangle \in I_{m-N R((\exists y) \varphi)}$ with $p \subseteq p^{\prime}$, $r \subseteq r^{\prime}$ and such that $\left\|(\exists y) \varphi\left(d_{1}, \ldots, d_{k}\right)\right\|_{\mathbf{M}}^{\boldsymbol{A}} \in \operatorname{dom}\left(p^{\prime}\right)$ and

$$
p^{\prime}\left(\left\|(\exists y) \varphi\left(d_{1}, \ldots, d_{k}\right)\right\|_{\mathbf{M}}^{\boldsymbol{A}}\right)=\left\|(\exists y) \varphi\left(r^{\prime}\left(d_{1}\right), \ldots, r^{\prime}\left(d_{k}\right)\right)\right\|_{\mathbf{N}}^{\boldsymbol{B}} .
$$

By the (Forth Property II) of Definition 6 , if $a=\left\|(\exists y) \varphi\left(d_{1}, \ldots, d_{k}\right)\right\|_{\mathbf{M}}^{\boldsymbol{A}}$, then there is a pair $\left\langle p^{\prime}, r\right\rangle \in I_{m-1}$ with $a \in \operatorname{dom}\left(p^{\prime}\right)$ and $p \subseteq p^{\prime}$.

Let $b=\left\|(\exists y) \varphi\left(r\left(d_{1}\right), \ldots, r\left(d_{k}\right)\right)\right\|_{\mathbf{N}}^{B}$, we show that $p^{\prime}(a)=b$. By (Back Property II) of Definition 6, there is a pair $\left\langle p^{\prime \prime}, r\right\rangle \in I_{m-2}$ with $b \in \operatorname{rg}\left(p^{\prime \prime}\right)$, and $p^{\prime} \subseteq p^{\prime \prime}$. Let $b_{0} \in A$ be such that $p^{\prime \prime}\left(b_{0}\right)=b$.

We show that $p^{\prime}(a) \geq b$ and $b_{0} \geq a$. As a consequence $p^{\prime}(a)=b$ and the partial isomorphism $\left\langle p^{\prime}, r\right\rangle$ will have the desired properties, with $\left\langle p^{\prime}, r\right\rangle \in$ $I_{m-N R((\exists y) \varphi)}, p \subseteq p^{\prime},\left\|(\exists y) \varphi\left(d_{1}, \ldots, d_{k}\right)\right\|_{\mathbf{M}}^{\boldsymbol{A}} \in \operatorname{dom}\left(p^{\prime}\right)$, and

$$
p^{\prime}\left(\left\|(\exists y) \varphi\left(d_{1}, \ldots, d_{k}\right)\right\|_{\mathbf{M}}^{\boldsymbol{A}}\right)=\left\|(\exists y) \varphi\left(r\left(d_{1}\right), \ldots, r\left(d_{k}\right)\right)\right\|_{\mathbf{N}}^{\boldsymbol{B}} .
$$

We prove that $p^{\prime}(a) \geq b$, the proof that $b_{0} \geq a$ is analogous. For every $e \in N$, we show that $p^{\prime}(a) \geq\left\|\varphi\left(e, r\left(d_{1}\right), \ldots, r\left(d_{k}\right)\right)\right\|_{\mathbf{N}}^{B}$, and since $b$ is the supremum of all these values, we will obtain that $p^{\prime}(a) \geq b$. Let $e \in N$, by the (Back Property I) of Definition 6, there is a partial mapping $r_{e}$ such that $e \in \operatorname{rg}\left(r_{e}\right), r \subseteq r_{e}$, and $\left\langle p^{\prime \prime}, r_{e}\right\rangle \in I_{m-3}$. Let $d \in M$ be such that $r_{e}(d)=e$. By the inductive hypothesis, there is a pair $\left\langle p_{0}, r_{0}\right\rangle \in I_{m-3-N R(\varphi)}$ with $p^{\prime \prime} \subseteq p_{0}, r_{e} \subseteq r_{0}$, such that $\left\|\varphi\left(d, d_{1}, \ldots, d_{k}\right)\right\|_{\mathbf{M}}^{\boldsymbol{A}} \in \operatorname{dom}\left(p_{0}\right)$, and

$$
p_{0}\left(\left\|\varphi\left(d, d_{1}, \ldots, d_{k}\right)\right\|_{\mathbf{M}}^{\boldsymbol{A}}\right)=\left\|\varphi\left(e, r_{0}\left(d_{1}\right), \ldots, r_{0}\left(d_{k}\right)\right)\right\|_{\mathbf{N}}^{\boldsymbol{B}} .
$$

Since $a=\left\|(\exists y) \varphi\left(d_{1}, \ldots, d_{k}\right)\right\|_{\mathbf{M}}^{\boldsymbol{A}}$, we have:

$$
p^{\prime}(a)=p_{0}(a) \geq p_{0}\left(\left\|\varphi\left(d, d_{1}, \ldots, d_{k}\right)\right\|_{\mathbf{M}}^{\boldsymbol{A}}\right)=\left\|\varphi\left(e, r_{0}\left(d_{1}\right), \ldots, r_{0}\left(d_{k}\right)\right)\right\|_{\mathbf{N}}^{\boldsymbol{B}},
$$

${ }_{445}$ and thus $p^{\prime}(a) \geq b$. The case of the universal formulas is analogous. Finally, by Remark 3, and (**), it follows that $\langle\boldsymbol{A}, \mathbf{M}\rangle \equiv_{n}\langle\boldsymbol{B}, \mathbf{N}\rangle$. 
Example 15 shows that the converse implication of Theorem 24 is not true in general.

\section{Generalization and conclusions}

The previous sections have been presented as an investigation of conditions for elementary equivalence of fuzzy first-order models, understood as the semantics of first-order fuzzy logics built over propositional logics extending the uninorm logic UL. Such formulation is convenient because it already covers (the models of) the majority of first-order fuzzy logics studied in the literature and already allows to find a wealth of examples and counterexamples to illustrate the merits and limitations of our results.

However, a careful inspection of the proofs of the theorems that we have obtained reveals that, actually, we have not used many properties of the underlying logical and algebraic framework. Observe that from all the connectives present in UL and its extensions only three have been really used: the constant $\overline{1}$ that determines the filter that allows to define logical consequence, and the implication $\rightarrow$ and conjunction $\wedge$ that determine the order relation in the algebras that is essential to define the filter and the semantics of quantified formulas. Other connectives, commutativity, residuation, and prelinearity have actually played no role.

Therefore, the whole paper could be rewritten, while keeping the same proofs, for the much wider framework of non-classical first-order algebraizable logics proposed in [9] based on algebraizable propositional logics (in the sense of [3]) which includes the majority of non-classical systems deeply studied in the literature. In fact, we have used this general framework in our previous investigation, closely related to the present one, of Löwenheim-Skolem theorems for non-classical logics [15].

In any case, either in the fuzzy formulation that we have chosen or in the possible general framework just mentioned, this paper has presented some advances in the central topic of elementary equivalence, that is, the classification 
of fuzzy structures according to their first-order theory. We have shown how the classical definition of elementary equivalence splits into three non-equivalent notions for non-classical logics. Using first a translation to classical two-sorted structures and later directly using non-classical structures, we have found backand-forth conditions that turn out to be sufficient, but not necessary, for the defined notions of elementary equivalence. We have illustrated their use with natural examples of fuzzy structures.

At this point there are several directions that should be pursued in further investigations. For instance, it would be desirable to find other usable criteria actually equivalent to elementary equivalence; a possibility to be explored would be back-and-forth conditions based on some generalized notion of EhrenfeuchtFraïssé games. A good understanding of elementary equivalence and back-andforth systems will lead to study other important issues such as categoricity, definability, saturation, and quantifier elimination.

\section{Acknowledgments}

We are very grateful to the anonymous referees for their helpful remarks. This project has received funding from the European Union's Horizon 2020 research and innovation programme under the Marie Sklodowska-Curie grant agreement No 689176 (SYSMICS project). Dellunde and García-Cerdaña are supported by the project RASO TIN2015-71799-C2-1-P (MINECO/FEDER). Dellunde is also supported by the grant 2014SGR-118 from the Generalitat de Catalunya. García-Cerdaña is also supported by the grant 2014SGR-788 from the Generalitat de Catalunya. Noguera is supported by the joint project of Austrian Science Fund (FWF) I1897-N25 and Czech Science Foundation (GAČR) GF15-34650L.

\section{References}

[1] J. Barwise, Back and forth through infinitary logic, in: Studies in Model Theory, Vol. 8 of MAA Studies in Mathematics, M. Morley, 1973, pp. 5-34. 
[2] R. Bělohlávek, Fuzzy Relational Systems: Foundations and Principles, Vol. 20 of IFSR International Series on Systems Science and Engineering, Kluwer and Plenum Press, New York, 2002.

[3] W. J. Blok, D. L. Pigozzi, Algebraizable Logics, Vol. 396 of Memoirs of the American Mathematical Society, American Mathematical Society, Provi-

a dence, RI, 1989, freely downloadable from http://orion.math.iastate. edu/dpigozzi/

[4] X. Caicedo, The strength of Łukasiewicz predicate logic, ManyVal-2015 Book of abstracts, pp. 8-9, Les Diablerets, Switzerland (2015).

[5] C. C. Chang, H. J. Keisler, Model theory, Vol. 73 of Studies in Logic and the Foundations of Mathematics, North-Holland, Amsterdam, 1973.

[6] C. C. Chang, H. J. Keisler, Continuous Model Theory, Vol. 58 of Annals of Mathematical Studies, Princeton University Press, Princeton, 1966.

[7] P. Cintula, F. Esteva, J. Gispert, L. Godo, F. Montagna, C. Noguera, Distinguished algebraic semantics for t-norm based fuzzy logics: Methods and algebraic equivalencies, Annals of Pure and Applied Logic 160 (1) (2009) 53-81.

[8] P. Cintula, C. G. Fermüller, P. Hájek, C. Noguera (Eds.), Handbook of Mathematical Fuzzy Logic (in three volumes), Vol. 37, 38, and 58 of Studies in Logic, Mathematical Logic and Foundations, College Publications, 2011 and 2015 .

525

[9] P. Cintula, C. Noguera, A Henkin-style proof of completeness for first-order algebraizable logics, Journal of Symbolic Logic 80 (1) (2015) 341-358.

[10] P. Cintula, C. Noguera, N. J. Smith, A logical framework for graded predicates, in: 6th International Conference on Logic, Rationality and Interaction - LORI 2017, 2017, p. In press. 
[11] P. Dellunde, Preserving mappings in fuzzy predicate logics, Journal of Logic and Computation 22 (6) (2012) 1367-1389.

[12] P. Dellunde, Revisiting ultraproducts in fuzzy predicate logics, Journal of Multiple-Valued Logic and Soft Computing 19 (1) (2012) 95-108.

[13] P. Dellunde, Applications of ultraproducts: from compactness to fuzzy elementary classes, Logic Journal of the Interest Group of Pure and Applied Logic 22 (1) (2014) 166-180.

[14] P. Dellunde, F. Esteva, On elementary equivalence in fuzzy predicate logics, Archive for Mathematical Logic 52 (2013) 1-7.

[15] P. Dellunde, À. García-Cerdaña, C. Noguera, Löwenheim-skolem theorems for non-classical first-order algebraizable logics, Logic Journal of the IGPL 24 (3) (2016) 321-345.

[16] A. Di Nola, G. Gerla, Fuzzy models of first-order languages, Zeitschrift für Mathematische Logik und Grundlagen der Mathematik 32 (19-24) (1986) 331-340.

[17] H.-D. Ebbinghaus, J. Flum, W. Thomas, Mathematical Logic, SpringerVerlag, New York, 1984.

[18] A. Ehrenfeucht, An application of games to the completeness problem for formalized theories, Fundamenta Mathematicae 49 (2) (1961) 129-141.

[19] J. Fodor, M. Roubens, Fuzzy Preference Modelling and Multicriteria Decision Support, Vol. 14 of Theory and Decision Library, Series D, Kluwer Academic Publishers, Dordrecht, 1994.

[20] R. Fraïssé, Sur quelques classifications des relations, basees sur des isomorphismes restreints. ii. applications aux relations d'ordre, et construction d'exemples montrant que ces classifications sont distinctes, Publications scientifiques de l'Université d'Alger 2 (1955) 273-295. 
[21] R. Fraïssé, Application des y-operateurs au calcul logique du premier echelon, Zeitschrift für mathematische Logik und Grundlagen der Mathematik 2 (1965) 76-92.

[22] P. Hájek, Metamathematics of Fuzzy Logic, Vol. 4 of Trends in Logic, Kluwer, Dordrecht, 1998.

[23] P. Hájek, P. Cintula, On theories and models in fuzzy predicate logics, Journal of Symbolic Logic 71 (3) (2006) 863-880.

[24] P. Hájek, L. Godo, F. Esteva, A complete many-valued logic with product conjunction, Archive for Mathematical Logic 35 (3) (1996) 191-208.

[25] F. Hausdorff, Grundzüge der Mengenlehre, Veit, Leipzig, 1914.

[26] W. Hodges, Model Theory, Vol. 42 of Encyclopedia of Mathematics and its Applications, Ox, Cambridge, 1993.

[27] E. V. Huntington, The continuum and other types of serial order, with an introduction to Cantor's transfinite numbers, Cambridge Mass, Harvard University Press, 1917.

[28] G. Metcalfe, F. Montagna, Substructural fuzzy logics, Journal of Symbolic Logic 72 (3) (2007) 834-864.

[29] J. N. Mordeson, P. S. Nair, Fuzzy graphs and fuzzy hypergraphs, Vol. 46 of Studies in Fuzziness and Soft Computing, Physica-Verlag, Heidelberg, 2000.

[30] P. Murinová, V. Novák, Omitting types in fuzzy logic with evaluated syntax, Mathematical Logic Quarterly 52 (3) (2006) 259-268.

[31] V. Novák, On the syntactico-semantical completeness of first-order fuzzy logic part I (syntax and semantic), part II (main results), Kybernetika 26 (1990) 47-66, 134-154. 
[32] V. Novák, I. Perfilieva, J. Močkoř, Mathematical Principles of Fuzzy Logic, Kluwer, Dordrecht, 2000.

[33] J. Pavelka, On fuzzy logic I, II, III, Zeitschrift für Mathematische Logik und Grundlagen der Mathematik 25 (1979) 45-52, 119-134, 447-464.

585

[34] A. Tarski, Der wahrheitsbegriff in den formalisierter sprachen, Studia Philosophica 1 (1936) 261-405.

[35] A. Tarski, R. L. Vaught, Arithmetical extensions of relational systems, Conmpositio Mathematica 13 (1957) 81-102. 